\title{
Norois
}

Environnement, aménagement, société

\section{Patrimoine et création dans la fabrique territoriale : l'estuaire ligérien ou la construction d'un territoire métropolitain.}

Heritage and the Making of an Urban Territory on the Loire Estuary

\section{Vincent Coëffé et Jean-René Morice}

\section{OpenEdition}

\section{Journals}

\section{Édition électronique}

URL : http://journals.openedition.org/norois/4746

DOI : $10.4000 /$ norois. 4746

ISBN : 978-2-7535-2918-2

ISSN : $1760-8546$

\section{Éditeur}

Presses universitaires de Rennes

\section{Édition imprimée}

Date de publication : 30 septembre 2013

Pagination : 77-88

ISBN : 978-2-7535-2883-3

ISSN : 0029-182X

\section{Référence électronique}

Vincent Coëffé et Jean-René Morice, « Patrimoine et création dans la fabrique territoriale : l'estuaire ligérien ou la construction d'un territoire métropolitain. », Norois [En ligne], 228 | 2013, mis en ligne le 30 septembre 2015, consulté le 21 avril 2019. URL : http://journals.openedition.org/norois/4746 ; DOI : $10.4000 /$ norois. 4746 

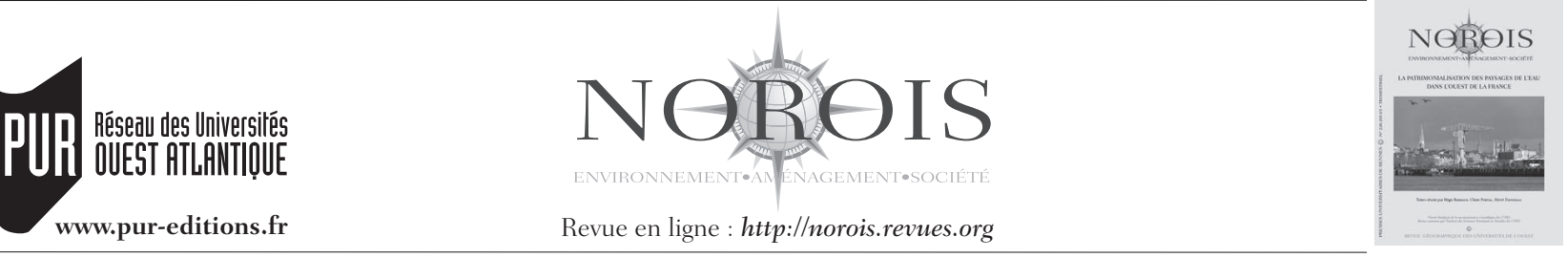

\title{
Patrimoine et création dans la fabrique territoriale : l'estuaire ligérien ou la construction d'un territoire métropolitain
}

\author{
Heritage and the Making of an Urban Territory on the Loire Estuary
}

Vincent CoËFFÉ*a, Jean-René MoricE

\footnotetext{
* Auteur correspondant

a, b PRES L'UNAM, UMR CNRS 6590 ESO-Angers, Université d’Angers (UFR ITBS), 7, allée François-Mitterrand

-49004 ANGERs (vincent.coeffe@univ-angers.fr) (jean-rene.morice@univ-angers.fr)
}

\begin{abstract}
Mots clés : Le patrimoine connaît depuis plusieurs décennies une extension de son champ et une redéfinition de ses formes. Alors que l'estuaire entre Nantes et Saint-Nazaire a longtemps été fragmenté, de nouvelles représentations ont émergé dans les dernières années qui en ont transformé le sens. Cet article cherche à montrer les différentes temporalités qui sont en jeu dans un processus de patrimonialisation à travers lequel certains acteurs tentent de produire un territoire. C'est en fait au croisement du tourisme, de l'art contemporain et du patrimoine que l'on peut saisir la métamorphose de l'estuaire. La patrimonialisation passe en effet ici par des acteurs engagés dans la production créative (l'art contemporain) et la valorisation touristique, l'opération Estuaire constituant un opérateur central dans la synergie mise en œuvre entre ces trois dimensions. Cette co-production, dont les filiations articulent « temps long » et « temps court », serait ainsi capable de fabriquer un territoire métropolitain.
\end{abstract}

Abstract: The concept of heritage has been widened and redefined for several decades by an extension of its field and a redefining of its forms. While the estuary between Nantes and Saint-Nazaire did not show unity for a long time, new representations have appeared within the last years which have transformed the sense of place. This article aims at showing the various temporalities which are at stake in the process of heritage making through which stakeholders try to make a territory. We can grasp the metamorphis of the estuary by looking at tourism, contemporary art and heritage practises. Indeed, heritage making is here carried out by stake involved in the creative production (the contemporary art) and tourism improvement with the Estuary event, essential operation that creates a synergy between these three dimensions. This collaboration, which articulates long term and short term, would be capable of producing a metropolitan territory.

Mots clés : patrimonialisation - création - réseau - territoire métropolitain - tourisme - estuaire - Nantes-Saint-Nazaire

Keywords : heritage making - creation - network - metropolitan territory - tourism - estuary - Nantes-Saint-Nazaire

Alors que le patrimoine a longtemps été associé au seul monument historique (et inversement), nous assistons depuis quelques décennies à une extension de cette catégorie qui intègre des œuvres toujours plus récentes, si bien que certains parlent même d'«inflation patrimoniale» (Heinich, 2009, p. 15).
Cet élargissement récent du champ patrimonial qui semble s'ancrer dans un temps toujours plus court (Lazzarotti et Violier, 2007), nous invite à creuser le problème de la dimension temporelle qui structure le processus de patrimonialisation. En effet, de nombreux travaux montrent que le patrimoine n'est 
pas un objet en soi mais une construction sociale et culturelle qui couple le temps et l'espace (Choay, 1992 ; Veschambre, 2005 ; Auduc, 2008). Le patrimoine est une réalité qui peut ainsi être saisie à travers un ensemble d'actions (la patrimonialisation) consistant en l'identification, la conservation et la transmission d'objets ou de pratiques incarnant une certaine représentation du passé. Ce processus s'apparente à une « opération intellectuelle, mentale et sociale qui implique des tris, des choix donc des oublis » (Lazzarotti, 2003).

Or, le patrimoine, longtemps concentré sur un objet ou un ensemble restreint d'objets s'est progressivement étiré pour recouvrir aujourd'hui des espaces de plus grande taille, dont les composants sont devenus hybrides, faits de culture mais aussi de nature. Cette extension prendrait sens dans un contexte de controverse : une contestation de la modernité qui passerait notamment par un engagement identitaire travaillé par la mondialisation. Les individus et les sociétés recomposeraient leurs identités, en lien avec l'accélération de l'effacement des traces du passé - qui se traduit dans le champ économique par la disqualification des «systèmes productifs d'antan et certains biens qu'ils livraient » (Di Méo, 2007, p. 6) - et la mondialisation productrice d'une mass culture. Cette dynamique générerait une certaine «frénésie patrimoniale », un investissement dans les valeurs culturelles héritées et ancrées dans le temps plus ou moins long des territoires (Di Méo, 2007, p. 10).

On le voit, la problématique du patrimoine semble articuler des temporalités paradoxales, obligeant à travailler des objets en tension. Sans cesse renouvelée, l'idée patrimoniale inciterait-elle finalement les individus et les groupes sociaux à être toujours plus créatifs, jusquà engager le mélange des genres qui passerait notamment par l'intrusion de l'art contemporain (Jimenez, 2005) et de l'événementiel (Morice et Violier, 2009)? Si la question du patrimoine est régulièrement explorée par les géographes, celle de son articulation avec la création contemporaine reste beaucoup plus discrète. Ces derniers, en dépit d'un certain " tournant culturel », ont longtemps prêté une attention toute relative à la « création artistique»(Grésillon, 2008). Enjeu de reconquête et de recomposition, l'art apparaît pourtant au regard des territoires comme une ressource innovante, un potentiel de développement important, source de nouvelles centralités à l'échelle des villes, qui tend à faire de l'approche géographique de l'art un objet de plus en plus investi par les chercheurs (Volvey, 2000 ; Gilabert, 2004 ; Boichot, 2010).

Dans cet article, nous proposons l'hypothèse suivante : la création contemporaine pourrait constituer un élément intégré au processus de patrimonialisation des objets spatiaux. Non seulement l'art contemporain contribuerait à faire émerger de nouvelles formes patrimoniales, mais il serait également un enjeu pour la fabrication de territoires. L'étude propose d'examiner, à partir du cas de l'estuaire ligérien, les conditions de développement et d'élaboration d'un nouveau patrimoine, selon une configuration qui mêle des œuvres contemporaines à des héritages industriels et portuaires. Ce processus pose la question du temps comme dimension à travers laquelle les individus en société donnent du sens à l'espace et produisent du territoire. L'espace retenu, l'estuaire de la Loire entre Nantes et SaintNazaire a été questionné par l'analyse des discours des acteurs, saisis à travers des supports de communication ou des publications, afin de compléter l'enquête réalisée durant l'été 2009 auprès du public fréquentant la manifestation d'art contemporain «Estuaire Nantes-Saint-Nazaire 2009 », le Château des ducs de Bretagne de Nantes et les Machines de l'Île de Nantes (Morice, 2009). Elle a permis de mieux comprendre les temporalités de la patrimonialisation des lieux. Dans quelle mesure une manifestation de ce type met-elle en évidence l'articulation du temps long et du temps court et contribue-t-elle au processus de mise en patrimoine de l'espace ligérien? Comment cette « fabrique du patrimoine » (Heinich, 2009), adossée à la création contemporaine, sert-elle de ressource pour produire du territoire? En quoi le tourisme constitue-t-il un levier pour déclencher ou catalyser ce processus?

\section{L'ESTUAIRE LIGÉRIEN : UN ESPACE FRAGMENTÉ}

Le vocable « estuaire » renvoie spontanément à une réalité biophysique, à cette partie d'un fleuve appelée l'embouchure où l'effet de la mer commence à être perceptible. Pour autant, cette définition à minima ne suffit pas à unifier l'estuaire ligérien, certaines réalités ayant même tendance ici à créer de la disjonction. 


\section{Une qualite des lieux tendue entre l'« industriel » et le « naturel»}

Soumise aux marées, la partie aval du fleuve qu'est l'estuaire, se voit caractérisée par des phénomènes physiques particuliers de type «bouchon vaseux » et «bouillonnement des flots» (Brunet, Ferras et Théry, 1993, p. 197). Cela passe par une forte charge de matières en suspension, « la crème de vase », où il est aisé de retrouver « des accumulations de pigments de dégradation, de traces de contamination fécale et divers micropolluants, dont l'expulsion en mer ne se produit qu'en période de crue » (Pirazzoli, 1997, p. 69). Malgré ces indices pouvant porter des valeurs négatives, les sociétés humaines ont très tôt cherché à maîtriser ce " milieu » ayant toutefois « l'avantage de permettre aux navires de mer de remonter loin à l'intérieur des terres ». Si « l'extrémité intérieure de l'estuaire » est généralement marquée «par un site classique de port, associé à une ville disposant d'un large arrière-pays », "l'extrémité aval » a, quant à elle, « souvent vu apparaître des avant-ports, exigés par l'accroissement de la taille des navires » (Brunet et al., 1993, p. 197). De cette empreinte humaine et cette interrelation portuaire, résulte une « exploitation de l'estuaire » qui peut se traduire par l'émergence de marqueurs industriels (Bavoux, 1997, p. 85).

Cette définition de la notion d'estuaire que construisent les géographes au cours des années 1990, se rapporte plutôt bien à l'espace étudié qui s'étend entre Nantes et Saint-Nazaire. Loin d'être représenté comme un objet suscitant et incarnant des valeurs esthétiques, l'estuaire Nantes - SaintNazaire est surtout resté un espace fonctionnel voué majoritairement aux échanges économiques et à la production industrielle (figure 1 - planche I). Généralement décrite comme polluée, cette Loire inférieure fut qualifiée par Julien Gracq d'estuaire « au ciel bas, encrassé de fumées jaunes et grises » (Gracq, 1985, p. 188). Ancrée dans le paysage, l'activité industrielle fait de l'estuaire une « zone sensible » à tous points de vue. Vingt-trois entreprises sont des sites industriels classés Seveso, présentant des risques majeurs au regard des quantités et des types de produits dangereux qu'elles accueillent. Pour six d'entre elles, toutes situées le long de l'estuaire, elles connaissent même un classement en seuil haut.
Si aujourd'hui l'espace est toujours fortement imprégné par l'industrie, une campagne, s'enchâsse en même temps au cour de cette "substance usinière » (Gracq, 1985, p. 187). Sur les bords de ces agrégats industrialo-portuaires se déploient, de part et d'autre du fleuve, de vastes zones humides, pour certaines difficilement pénétrables, soumises aux flux des marées, constituées de marais, de roselières, de vasières ou encore de prairies salées inondées quelques jours dans l'année. Entre le fleuve et ces zones humides, l'eau tient une place prépondérante. C'est bien face au risque d'étalement et de développement constant du grand port maritime Nantes-Saint-Nazaire que ces zones humides, définies comme "naturelles » dans un contexte pourtant fortement industrialisé, ont fait l'objet de strictes procédures de protection. Certaines furent ainsi classées en "Zone de Protection Spéciale» et «Zone Spéciale de Conservation », elles-mêmes incluses dans une «Zone Naturelle d'Intérêt Ecologique Faunistique et Floristique » (figure 2 - planche II). Plusieurs réserves de chasse existent et garantissent la reproduction et l'hivernage de l'avifaune. Ce souci de préservation des lieux s'inscrit depuis 1994 dans un programme nommé Plan Loire Grandeur Nature intégrant notamment le Groupement d'Intérêt Public Loire Estuaire qui propose à travers un Comité Estuaire de mener une approche prospective et raisonnée de l'espace estuarien.

\section{Une situation périphérique marquée par de la discontinuité}

Alors que la partie maritime de l'estuaire constitue bien une interface, sa partie amont se trouve davantage projetée en marge par les représentations d'acteurs externes. Si Nantes peut en effet être appréhendée comme la pointe avancée du paysage estuarien, la ville marque en même temps la fin de la Loire mise en tourisme et reste à l'écart du périmètre du Val de Loire, site Unesco (dont la limite aval est positionnée à Chalonnes-sur-Loire, près d'Angers). Cette labélisation a été attribuée en 2000 par l'Unesco, au titre de ses paysages culturels associés essentiellement à l'image monumentale des châteaux (figure 3 - planche III). Les valeurs des héritages industriels ont été tardivement intégrées par les acteurs institutionnels de la valorisation patri- 
moniale (Béghain, 1998). À l'exemple du canal de la Martinière, l'estuaire compte tout un ensemble de lieux et de biens autrefois liés à l'activité industrielle et maritime, aujourd'hui désaffectés et pour beaucoup à l'état de vestiges. Seuls quelques-uns sont protégés au titre de la loi de 1913 et ont fait l'objet d'un classement sur la liste des Monuments historiques, à l'exemple de la tour à plomb de Couëron.

Par ailleurs, si Nantes est l'ancienne capitale des ducs de Bretagne, la métropole est aujourd'hui la capitale administrative de la région des Pays de la Loire. Nantes a fondé son développement sur l'activité commerciale au XVIII ${ }^{\mathrm{e}}$ siècle puis sur l'industrie au XIX ${ }^{\text {e }}$ siècle (Rapetti, 2004). Cette activité florissante a été mise à mal par la crise des années 19701980 qui s'est traduite par la fermeture des chantiers navals en 1987. L'obsolescence de ces activités économiques s'est traduite par certaines représentations urbaines, à tel point que certains ont parlé de « ville endormie » (Grandet et al., 2010), alors même que Nantes bénéficie depuis les années 1990, d’un « indiscutable succès médiatique et [...] caracole, dans les multiples classements de villes opérés par la presse, parmi le peloton de tête des villes où il fait bon vivre » (Fritsch, 2006).

Cela dit, la fabrication d'une cohérence métropolitaine à l'échelle de l'estuaire reste inachevée. S'il existe une «forte indépendance liée à la construction navale des Chantiers de l'Atlantique, à l'axe industriel et logistique de l'estuaire, [...] Nantes et Saint-Nazaire sont clairement disjointes [...]. Chacune dispose de son propre bassin de vie même s'il existe un espace interstitiel multipolarisé entre les deux aires urbaines : il n'existe pas un marché mais bien deux marchés du travail » (Fritsch, 2006). De plus, les mobilités récréatives depuis l'agglomération nantaise sont polarisées par les communes du littoral (La Baule, Pornic...) plus que par SaintNazaire. La création en 2003 d'un SCOT (Schéma de COhérence Territoriale) métropolitain n'a par ailleurs pas encore pleinement généré de coalescence entre les lieux. Le fait que la communauté urbaine de Nantes se soit baptisée Nantes-métropole au moment où l'échelle métropolitaine visée par le nouvel outil d'aménagement devait englober les deux agglomérations, en constitue de ce point de vue un des signes les plus éclairants. Au regard des lignes de fragmentation qui structurent en partie l'espace estuarien, on peine donc à voir émerger les éléments susceptibles de l'organiser en territoire, lequel suppose des lieux entretenant des relations au travers d'acteurs qui y puisent et y projettent des valeurs collectives produites dans l'épaisseur temporelle (Di Méo, 1998).

\section{L'ÉVÉNEMENT ESTUAIRE : LE TEMPS DE LA BIFURCATION}

De ce point de vue, la biennale d'art contemporain Estuaire Nantes-Saint-Nazaire, aurait-elle permis d'opérer une bifurcation en contribuant à la mise en réseau des lieux, surtout si cette action culturelle était « instrumentalisé afin de créer une image qui dépasse le temps de l'événement » (Barthon et al., 2007)?

\section{Estuaire : une suite de biennales pour l'émergence d'un reseau}

Ce projet de biennale a été imaginé par Jean Blaise qui, déjà au début des années 1980, avait mis en place à Nantes un Centre de Recherche pour le Développement Culturel et une maison de la culture. C'est dans le cadre de cette dernière structure, qui devait être cofinancée à parts égales par l'État et les collectivités locales, que Jean Blaise se rapprocha de Jean-Marc Ayrault, alors maire de Saint-Herblain, les deux acteurs imaginant la création d'une scène nationale itinérante, capable d'accueillir les grandes compagnies de l'époque. Dès ce moment donc, émerge un changement d'échelle opéré par un projet culturel. Devenu directeur du Lieu unique $^{1}$, Jean Blaise cherche en même temps à diffuser la création artistique hors les murs. Mise en œuvre en 2007, la première édition d'Estuaire a toutefois alimenté une certaine controverse, notamment à propos de son coût ( 7,5 millions d'euros, dont 2 millions financés par des entreprises privées, le reste relevant des collectivités territoriales essentiellement) jugé exorbitant par certains nantais. La critique portait également sur les choix effectués en termes de proposition artistique. Un débat a même été co-organisé par la revue Place publique

\footnotetext{
1. Le Lieu unique, inauguré à la fin de l'année 1999 et installé à Nantes dans un ancien bâtiment de l'usine LU, mêle aujourd'hui au sein d'un même site, une scène nationale, des espaces d'exposition, un restaurant, une librairie, une crèche et un hammam.
} 
et Nantes, culture et patrimoine, au moment du lancement de l'opération².

Pour autant, la biennale a connu une nouvelle occurrence en 2009 et l'édition 2012 marque l'aboutissement du projet artistique. De ces trois moments créatifs a émergé un réseau d'œuvres in situ, disséminé entre Nantes et Saint-Nazaire, sur les 60 kilomètres de part et d'autre des deux rives. Si certaines créations ont été conçues pour façonner le paysage durant le temps éphémère de l'événement, d'autres l'ont été selon une orientation « durable » en déployant à l'échelle de l'estuaire un parcours pérenne accessible au public. La singularité de l'opération tient à la constitution d'une collection contemporaine se rapprochant d'un «musée à ciel ouvert ${ }^{3}$ invitant à venir découvrir, au moyen de l'art, un espace jusque dans ses interstices. La troisième biennale propose de nouveaux contenus culturels à l'exemple de la «Ville renversée par l'art» : ici un ensemble de jalons artistiques et de patrimoines revisités par l'art, structure, à travers l'espace public nantais, un parcours qui se veut « insolite » et « festif ». De manière exemplaire, la proposition prévoit l'accès, après plus de 10 ans de fermeture, du $32^{\mathrm{e}}$ étage de la tour de Bretagne, aménagé par le plasticien Jean Julien en café œuvre d'art représentant le nid d'un héron. Annoncé comme l'un des futurs hauts-lieux touristiques de la ville, cette terrasse panoramique sécurisée offre un large point de vue sur Nantes et son estuaire, à près de 150 mètres d'altitude. Cette mise en regard qui placerait chaque visiteur en position surplombante (dominante), pourrait alors favoriser l'appropriation symbolique de l'espace, Nantes devenant le lieu à partir duquel se déploierait une aire métropolitaine incorporée à travers la vue.

Cette dialectique de la mobilité et de l'ancrage peut se lire également à travers les choix artistiques, lesquels composent pleinement avec les lieux. À chaque site, une vingtaine au total, est associé un artiste de stature internationale chargé de concevoir une ouvre en résonance étroite avec la qualité du lieu : Felice Varini (Suisse) et sa «Suite de triangles » de couleur rouge qui se déploie sur $2 \mathrm{~km}$ dans le port de Saint-Nazaire, Erwin Wurm (Autrichien) et son voilier déformé de 9 mètres

2. [http://revue-placepublique.fr/Sommaires/Sommaires/Articles/estuaire07. htm].

3. [wow nantes-tourisme.com].
« Misconceivable », installé au Pellerin à l'entrée du canal de la Martinière, Tatzu Nishi (Japonais) et sa «Villa Cheminée » perchée à quinze mètres de hauteur à Bouée à proximité de la centrale EDF de Cordemais ou encore Tadashi Kawamata (Japonais) et son "Observatoire » à Lavau-sur-Loire qui, au milieu des roselières, offre un cheminement en bois de 800 mètres et du haut d'une plateforme de 6 mètres, une perspective plongeant vers le fleuve. À compter de 2012, une trentaine d'œuvres forment ainsi un réseau de marqueurs paysagers construisant un nouvel espace de circulation à l'échelle de l'estuaire (figure 4 - planche IV).

L'estuaire se voit ainsi saisi dans une nouvelle trame géographique avec les propositions d'artistes étrangers qui projettent l'espace estuarien dans le Monde. Les œuvres ont pour fonction de révéler les lieux dans lesquels elles émergent, jusqu'à en faire de quasi hauts-lieux à visiter. Lors de l'enquête effectuée en 2009 auprès des publics ${ }^{4}$ (Morice, 2009), les visiteurs internationaux représentaient malgré tout une part encore très minoritaire : à peine $3 \%$ des visiteurs, venant pour près d'un tiers de Belgique. La Loire-Atlantique restait la principale filière de provenance des visiteurs, plus de $59 \%$ du total, reléguant les touristes vers une position secondaire pour le moment. Plus globalement, la stratégie engagée par les villes de Nantes et de Saint-Nazaire correspond à un projet politique. Le principe consiste à faire exister à travers l'art et sa médiatisation, un futur territoire métropolitain ligérien de rayonnement européen de près d'un million d'habitants.

\section{Estuaire : un événement entre patrimoine et création}

Depuis une vingtaine d'années, les acteurs du patrimoine tendent à s'ouvrir à la création (Morice, 2011), notamment à des artistes plasticiens (Buissart, 2009), de l'emballage du Pont Neuf à Paris par Christo (1985) aux expositions de Jeff Koons (2008) et Takashi Murakami (2010) au château de Versailles, en passant par les colonnes de Daniel Buren (1986) installées dans la cour du Palais Royal. Ce couplage d'objets incarnant l'authenticité sur le temps long avec des œuvres contemporaines asso-

4. 2500 questionnaires ont été traités ici. 
ciées à la modernité n'est pas sans produire de la controverse (Le Monde, 2 octobre 2010), ces dernières étant régulièrement suspectées de déprécier les biens patrimoniaux en fissurant la continuité temporelle. Cela dit, les configurations articulant œuvres «traditionnelles » et « créatives » ne sont pas tout à fait nouvelles, participant de la vie artistique et de ses avant-gardes qui ne le sont qu'à un moment donné, situé dans un contexte culturel et social produisant des valeurs plus ou moins dissonantes. Mais cette controverse peut être amplifiée lorsque ces œuvres font irruption dans des territoires improbables.

Quelle que soit l'installation ou la sculpture à découvrir, elle propose systématiquement une relecture du lieu investi. Il en est ainsi de cette centrale à béton à Trentemoult près de Rezé, mise à l'arrêt en 2005 et transformée en 2009 par Roman Signer en gigantesque «Pendule ». L'intervention se veut minimaliste : un grand pendule de 7 mètres accroché au bâtiment bat inexorablement le temps, marquant pour l'artiste la lente déchéance des éléments au rythme immuable et incessant du fleuve. À partir de cette matière détournée, la découverte du lieu s'appuie à la fois sur l'activité de cette ancienne centrale à béton, témoin industriel de la construction des ponts de Cheviré et de Pirmil à Nantes et sur sa localisation, celle d'un ancien village de pêcheurs et de cap-horniers de bord de Loire. Pour ce qui est de la dimension artistique, la création sous-tend la conception d'une valeur supérieure de l'art (Heinich, 1993), dans cette transformation voire transfiguration du non-art en art. Le geste créatif s'inscrit dans un processus qualifié "d'artification » où il s'agit «de requalifier les choses et de les anoblir : l'objet devient œuvre, le producteur devient artiste, la fabrication création, les observateurs un public » (Shapiro, 2004, p. 2). Plus encore, c'est l'ensemble du lieu qui se voit concerné par une modification de sens en référence au concept « d'artialisation » développé par Alain Roger (1997) pour qui l'intervention artistique (l'artiste et son regard) participerait à donner à un lieu une qualité esthétique capable de le faire passer du statut de "pays » à celui de «paysage » (alors associé à la notion de «beau »). Lacte de photographier les œuvres et l'environnement qui en constitue l'écrin, favoriserait la transformation symbolique de l'espace par son « artialisation », et contribuerait à amplifier la valeur esthétique du lieu.
Certaines œuvres de l'estuaire se voient ainsi saisies par et dans un réseau dense d'images, parmi lesquelles figurent les photographies des visiteurs, qui contribuent à en faire des icônes nouvelles du territoire estuarien.

Par ailleurs, le choix de nommer l'événement en convoquant un toponyme peut être interprété comme une démarche performative (Austin, 1970), les actes de langage contribuant à faire, et plus particulièrement ici à produire du territoire. C'est à travers le passage d'une terminologie géographique, «l'estuaire », à celle d'une marque de destination, «l'Estuaire », que s'écrit désormais le territoire. L'estuaire devient par la majuscule attribuée à la performance artistique, un quasi personnage géographique saisi par une intrigue, une «narration » qui crée l'histoire du territoire. Puisque l'estuaire pouvait faire l'objet de représentations contradictoires susceptibles de générer de la discontinuité voire de la fragmentation dans le territoire, il devenait éventuellement possible d'en faire advenir l'identité et la cohérence par un événement qui présente un ancrage géographique, à la fois immatériel par le toponyme "Estuaire » et matériel par des productions artistiques enchâssées dans l'espace. C'est d'autant plus vrai que la mise en scène des œuvres est d'emblée conçue selon un agencement invitant au parcours, donc à la mise en relation des lieux dans lesquels sont ancrées les productions artistiques. D'où le choix aussi de garantir la pérennité de certaines œuvres afin de proposer de nouvelles prises pour l'interprétation de l'espace estuarien. L'appel au patrimoine légitime, le monument, peut alors être plus facilement mobilisé, métamorphosant au passage la production contemporaine en y injectant une épaisseur temporelle, dont elle pouvait être dépourvue, pour devenir, aux dires de Jean Blaise, une sorte de «monument dispersé » le long de la Loire. La dilatation du temps peut se lire aussi à travers certaines œuvres qui cherchent à représenter le passé, comme c'est le cas des «Anneaux » de Daniel Buren et Patrick Bouchain, à la pointe de l'île de Nantes, dont le sens renvoie à l'héritage encombrant de la traite négrière dans l'histoire de l'estuaire ligérien. Cette œuvre contemporaine constituée d'une enfilade d'anneaux offrant à la perception un nouveau regard sur le paysage urbain, figure l'enchaînement des esclaves, et noue ainsi le présent et le passé de Nantes. La signification de 
l'œuvre ne se donne pas forcément dans l'immédiateté, et en tant qu'objet symbolique, elle active la commémoration d'un temps révolu qui aurait pu disparaitre dans l'oubli, du fait de l'absence de traces (d'héritages matériels) surtout liées à l'histoire de la traite qui font depuis les années 1980 notamment, l'objet d'un travail de mémoire de la part d'associations locales. Ainsi, les «Anneaux » ne sont pas sans faire échos à l'association Les anneaux de la mémoire créée dans le sillage d'un colloque qui s'était tenu en 1985 à l'université de Nantes sur le thème de la traite négrière et de l'esclavage. L'œuvre fonctionne donc ici comme un marquage temporel, chargé de faire advenir une mémoire en construction, portée par le projet métropolitain.

\section{VERS UN TERRITOIRE MÉTROPOLI- TAIN : NANTES-SAINT-NazAIrE}

La compétition territoriale exacerbée dans les dernières décennies entre les métropoles, a redéfini la configuration des projets urbains, lesquels font plus souvent appel à la culture et au tourisme comme moteurs de l'aménagement. Si l'opération Estuaire tend à « instaurer des effets durables », tout en permettant de «passer de l'échelle urbaine à l'échelle métropolitaine »(Gravari-Barbas, 2013, p. 89), c’est aussi parce que cet événement s'inscrit dans un projet de métropolisation plus global, tendant à faire converger différents acteurs impliqués aussi bien dans le champ de la gouvernance urbaine que dans ceux de la culture, du tourisme et de l'aménagement.

\section{La fabrique d'un territoire portée par un système d'acteurs}

L'estuaire de la Loire semble bien faire l'objet d'un projet de territoire, alors même que ses limites juridiques et géographiques sont parfois encore floues. Si l'art, de manière incontournable, participe à cette reconnaissance contemporaine du territoire, le jeu des acteurs dans la construction du territoire est également essentiel. À défaut d'une structuration unique, l'estuaire s'appuie aujourd'hui sur un système localisé d'acteurs institués, établi aussi «verticalement » (dans l'épaisseur du temps) et ayant participé dans le cadre de projets partagés à fabriquer le territoire. Ce poids des acteurs institutionnels peut alors favoriser une éventuelle appartenance estuarienne aujourd'hui matérialisée par des organisations nouvelles en développement. De manière complémentaire au SCOT, peut être cité le Grand port maritime de Nantes - Saint-Nazaire, préalablement dénommé Port autonome de Nantes - Saint-Nazaire de 1966 à 1991 puis Port Atlantique Nantes - SaintNazaire de 1991 à 2008, et dont la mission est de gérer l'activité maritime et portuaire de l'estuaire et les politiques publiques d'aménagement qui y sont associées. Cette construction métropolitaine est également passée par la progressive fusion au cours des années 1990 et 2000 des Chambres de Commerce et d'Industrie de Nantes et de SaintNazaire en une seule institution consulaire.

Cette fabrication du territoire ne peut être comprise non plus sans l'analyse du jeu culturel qui s'est constitué, plusieurs dizaines d'années avant l'opération Estuaire. Cette place de la création contemporaine dans les projets urbains de Nantes et de SaintNazaire est en fait amorcée dès les années 1980 avec Joël Batteux nouvellement élu à la mairie de SaintNazaire en 1983 et Jean-Marc Ayrault à Nantes en 1989. C'est bien à partir des années 1980, que la culture va constituer une composante importante des politiques urbaines nazairienne et nantaise, dans un contexte national où certains acteurs politiques, comme Jack Lang à plusieurs reprises ministre de la Culture, sont convaincus que l'élément culturel peut représenter un réel outil de « développement local ». Cette politique culturelle urbaine s'incarne à Saint-Nazaire par la mise en lumière du port par l'artiste plasticien Yann Kersalé. Très vite la dimension artistique se voit partie prenante du projet global de développement voulu par la ville. S'esquissent des lieux, le «Fanal », scène nationale lancée en 1991, le «Grand Café » en 1997, centre d'art contemporain, le « LiFE », Lieu International des Formes Émergentes, logé depuis 2007 dans l'Alvéole 14 de l'ancienne base sous-marine, des manifestations, le festival «Les Escales » depuis 1992, la « Grande Marée » en 2002, pique-nique géant dédié aux arts de la rue. La création contemporaine est retenue pour forger l'identité de la ville, réinterroger son présent, son devenir, sa mémoire et plus globalement accompagner sa transformation : « Le territoire à investir est bien celui de la ville et non pas seulement ceux des espaces réservés habituelle- 
ment aux expositions ${ }^{5}$. » Pour Nantes, cela passe en 1990 par l'installation de la compagnie Royal de luxe, spécialisée dans les arts de la rue. Cette même année, Jean Blaise est à l'initiative d'une nouvelle programmation culturelle à Nantes, le festival des Allumées, un événement qui permet à des artistes de plusieurs villes du Monde d'investir durant six nuits des friches industrielles de la ville, dont les anciennes usines LU. Cette gouvernance culturelle associant des élus, des professionnels et des artistes est loin de constituer au cours des années 1990 un modèle pour l'ensemble des municipalités à l'échelle nationale même si l'on retrouve par exemple cette configuration à Montpellier dès les années 1980 à travers la danse et la musique (Djian, 2005) ou plus récemment à Lille en 2004, à travers le label capitale européenne de la culture (Morice et Violier, 2009).

Que ce soit pour Nantes ou Saint-Nazaire, ces innovations culturelles accompagnent un processus de patrimonialisation en cours des villes et de renouvellement urbain, source de nouvelles centralités. Le projet de «Ville-Port » est imaginé dès le milieu des années 1990 à Saint-Nazaire, permettant à la ville de renouer avec ses héritages industriels et portuaires, l'ancienne base sous-marine passant d'une position de discontinuité dans la ville (entre le centre-ville et les bassins) à celle d'interface valorisée au travers de l'aménagement d'une rampe piétonne ouvrant un panorama sur le port et l'estuaire. La valorisation de l'activité navale y trouve même place depuis 2000, à travers la reconstitution de l'univers d'un paquebot transatlantique ouvert au public (Escal'Atlantic). Le récent programme de reconfiguration de l'île de Nantes ${ }^{6}$ cherche quant à lui à créer, à travers des opérations comme le quartier de la création et les Machines de lîle, une centralité complémentaire du centre historique, ce dernier étant patrimonialisé à travers ses Monuments historiques (123 biens classés ou inscrits selon la base Mérimée) et son secteur sauvegardé notamment. Les objets spatiaux produits par l'industrie ont été dans les dernières décennies intégrés à la catégorie du patrimoine au moment où les traces industrielles tendaient à être effacées par de profondes mutations économiques. Or, si

5. [www.mairie-saintnazaire.fr].

6. L'île de Nantes est un ancien pôle industrialo-portuaire situé au sud du centre-ville, et dont l'insularité résulte de l'unification progressive d'îles anciennement séparées par des bras de la Loire. la patrimonialisation opère des tris, entre destruction et conservation, "ce qui devient patrimoine, c'est ce qui est désaffecté et qui tend à disparaître » (Veschambre, 2005). Cette prise de conscience d'une possible disparition a pu susciter un intérêt patrimonial chez certains acteurs locaux à Nantes et Saint-Nazaire.

Dans un contexte contemporain de décentralisation qui affecte aussi les acteurs de la culture, le réseau local composé des milieux politiques, ouvriers, syndicaux, universitaires et patronaux, a fortement favorisé l'identification d'objets intégrés dans la « chaîne patrimoniale » (Heinich, 2009, p. 41), la patrimonialisation ne pouvant être déclenchée qu'à partir du moment où un objet est sorti de l'invisibilité pour entrer dans la machine de fabrication mémorielle. Si la prise en compte des héritages industriels a été initiée par Alain Chénard ${ }^{7}$, lequel a permis la réhabilitation de la Manufacture des Tabacs, Jean-Marc Ayrault lui a emboîté le pas dès son premier mandat, avec le souci de ménager la sensibilité des anciens de la Navale, lesquels ont fondé, en 1986, l'Association d'histoire de la construction navale à Nantes, afin d'œuvrer pour la préservation de la mémoire des chantiers. Cette posture s'est traduite en 1989 par le soutien de Jean-Marc Ayrault à la réhabilitation du bâtiment de la direction des Ateliers et Chantiers de la Loire (Nicolas, 2010). Ce dernier a été plus tard convaincu de la nécessité du rachat par la mairie de la grue Titan jaune, construite pour le compte des chantiers Dubigeon en 1954, et dotée d'une forte charge symbolique pour nombre d'acteurs locaux. Emblème de la mémoire industrielle des chantiers navals nantais, la valorisation patrimoniale de cette grue a finalement préparé le classement sur la liste des Monuments historiques d'une autre grue Titan (grise), acquise par le Port de Nantes-Saint-Nazaire en 1969, et qui figure aujourd'hui comme un élément structurant du paysage à l'extrémité ouest de l'île de Nantes. Le démontage de cette grue a d'ailleurs été abandonné au dernier moment en décembre 2004, grâce à la mobilisation des acteurs associatifs, ce qui montre que la dynamique patrimoniale n'est pas linéaire, et que le temps de la patrimonialisation peut très bien être discontinu (Barthon, 2005).

7. Chenard a été maire de Nantes de 1977 à 1983, ensuite, Michel Chauty (1983-1989) puis Jean-Marc Ayrault (1989-2012). 


\section{La production d'un territoire métropolitain au croisement de la culture et du tourisme}

À compter de 2010, l'opération Estuaire connaît un tournant important. Le regroupement de l'office de tourisme de Nantes Métropole, d'Estuaire Nantes - Saint-Nazaire et de la SEM Nantes Culture et Patrimoine en société publique locale " Le voyage à Nantes » se donne pour objectif de doter la métropole nantaise et plus largement l'estuaire d'une organisation capable de hisser le territoire parmi les premiers espaces touristiques d'Europe. C'est ainsi qu'a été imaginé le « Voyage à Nantes », structure lancée en janvier 2011, pilotée depuis sa création par Jean Blaise, également son instigateur. L'intégration «d'Estuaire » dans cet outil de développement touristique entérine aujourd'hui le projet de mise en tourisme des œuvres imaginé dès 2007. La biennale de 2007 proposait déjà un parcours singulier de présentation des œuvres, à travers une croisière sur l'estuaire, découverte toutefois controversée dans la mesure où les œuvres manquaient de visibilité depuis la Loire, alors même que la communication insistait sur la monumentalité de ces dernières. Ce dysfonctionnement avait été plus ou moins corrigé par la proposition d'autres modes de circuits de visite imaginés depuis les rives (Bossé, 2012, p. 54). L'ambition est de propager l'événement, de l'inscrire dans une temporalité qui garantisse une certaine continuité. D'où la construction dès 2007 par les organisateurs d'une trame narrative dans laquelle Estuaire est systématiquement projeté vers l'avenir pour devenir un parcours touristique durable.

L'initiative consistant à créer un outil comme le «Voyage à Nantes » doit être lu dans un contexte de mondialisation accélérée à travers laquelle les métropoles se livrent une compétition farouche pour la « lutte des places » (Lussault, 2009), pour l'intégration sur la scène mondiale par la fabrication et la valorisation d'un avantage compétitif. La production de singularité par l'appel à la création devient dans cette dynamique un élément de distinction (Florida, 2002). À Nantes, la notion de «ville créative " (Vincent, 2009) a été saisie par les édiles et les urbanistes de la Société d'Aménagement de la Métropole Ouest Atlantique (SAMOA) qui ont même imaginé un toponyme calé sur ce nou- veau paradigme : le « quartier de la création », situé sur l'île de Nantes, à l'endroit des anciennes usines Alstom. L'énonciation du projet par la SAMOA, le maître d'ouvrage, insiste sur l'ancrage et l'importance des « industries de la création » dans la définition du programme d'aménagement. L'idée est de réunir en un même lieu une grande diversité d'acteurs dans le domaine de la culture, du design, des arts de la scène, des arts visuels, de l'architecture et des médias, dans un principe proche de celui des pôles de compétitivité (Grandet et al., 2010). La Loire n'est pas ici conçue comme un simple intervalle, une discontinuité, mais comme un élément intégrateur du territoire : « La Loire devient le lien, le trait d'union entre le centre historique et l'île de Nantes et, plus largement, le symbole du nouvel élan de la métropole Nantes/Saint-Nazaire ${ }^{8}$. » Finalement, l'île de Nantes, qui joue comme une métonymie de ce qui se développe à l'échelle de l'estuaire, peut être lue comme un cluster culturel, dans lequel s'inscrit depuis le milieu des années 2000, sous la houlette de François Delarozière et de Pierre Oréfice anciens membres de Royal de Luxe, le projet monumental des Machines de l'Île, créations à la rencontre de différents univers hérités de Jules Verne ou de Léonard de Vinci. Selon les données produites en mars 2011, un million de visiteurs auraient franchi les portes des Machines de l'île depuis son ouverture en 2007 (source : Le Voyage à Nantes, 2012). L'emblème de ces mondes imaginaires reste l'Éléphant haut de 12 mètres, transportant sur son dos pour une promenade d'une vingtaine de minutes, quelques dizaines de passagers embarqués dans une nacelle. Selon l'enquête menée en $2009^{9}$ (Morice, 2009), plus de $30 \%$ du public interrogé avait d'ailleurs "visité » cette machine. Lî̀le de Nantes tient désormais une place de choix dans l'attractivité de la ville. Au regard des visuels dédiés à l'architecture du xxe siècle de la collection « Laissez-vous conter Nantes », du service "Ville d'art et d'histoire ", lîle concentre d'ailleurs la plus grande densité de lieux à visiter et bénéficie même d'une cartographie spécifique qui amplifie sa charge symbolique.

Cette articulation du tourisme et de la culture à l'échelle de l'estuaire est passée par une redéfinition du lien entretenu par le territoire avec la notion de

8. [www.iledenantes.com].

9. 500 questionnaires ont été traités ici. 
patrimoine mondial. Positionné en dehors du périmètre « paysage culturel du Val de Loire » (figure 4), l'estuaire connait depuis 2006 par l'intermédiaire de la ville de Nantes une éviction moins radicale. En effet, à travers le château des ducs de Bretagne, les acteurs nantais, ont participé à raccrocher partiellement l'estuaire au territoire du Val de Loire au moyen de l'opération des grands sites du Val de Loire. Portée par la Mission Val de Loire en charge du label, cette action de mise en réseau fédère depuis ses débuts 19 grands sites patrimoniaux du Val de Loire dont le château de Nantes. L'ambition alors affichée est de contribuer au renouvellement et à l'augmentation de la fréquentation touristique des sites de visite ligériens et de la destination Val de Loire dans son ensemble. À travers cette prise en compte du château des ducs de Bretagne, la Mission Val de Loire sort quelque peu de son périmètre d'intervention habituel, le territoire officiellement inscrit, en participant à faire reconnaître la Loire estuarienne nantaise comme site à part entière du Val de Loire. Cette intégration est renforcée par le tracé touristique de la "Loire à vélo », initiative interrégionale des Pays de la Loire et du Centre, qui offrira à terme près de 800 km d'itinéraires balisés le long de la Loire, jusqu'à son embouchure, de Nevers à Saint-Brévin-les-Pins. Quelque peu ignorée au lancement du projet, la partie ligérienne maritime se voit désormais pleinement intégrée au linéaire touristique sous le slogan « La Loire à vélo, c'est aussi l'estuaire! » (Envies de vacances en Pays de la Loire, 2012, p. 9).

\section{Conclusion}

La production territoriale de l'estuaire articule ainsi différentes échelles de temps et d'espace et s'appuie depuis les années 2000 notamment sur la culture en tant qu'élément susceptible d'alimenter la fabrique d'une identité géographique. La Biennale Estuaire, qui met en tension l'éphémère et le pérenne, a constitué de ce point de vue un catalyseur pour le changement d'échelle dans la mesure où l'estuaire ligérien était jusque récemment un espace fragmenté, peinant à produire une image cohérente capable de faire territoire, notamment à travers la production des outils d'aménagement. En devenant un espace parcouru, visitable, porté par un regard externe (les touristes entre autres), mais aussi interne (la société locale), l'estuaire devient un ensemble tissé de lieux. Éléments hier disgracieux, certains objets et lieux sont aujourd'hui présentés à la visite comme des réalités dignes d'intérêt, assimilées à de véritables marqueurs historiques et identitaires du territoire estuarien.

Mais la Biennale a été rendue possible par la constitution progressive d'un système d'acteurs à l'échelle métropolitaine, lesquels avaient plus tôt fait le choix d'intégrer la culture dans le projet urbain. Alors que Nantes et Saint-Nazaire ne se sont pas dotés d'un équipement culturel capable de fonctionner comme une icône urbaine par le travail d'architectes de renommée mondiale, à l'instar du Guggenheim de Franck Gehry à Bilbao (Coëffé, 2011), le patrimoine surgit ici d'un nouvel usage de lieux mis en culture et en réseau, la création permettant la valorisation de certains héritages. En retour, la création serait-elle progressivement intégrée dans la « chaîne patrimoniale » (Heinich, 2009)? Les «Anneaux » de Daniel Buren et Patrick Bouchain participent ainsi au travail sur une mémoire singulière, renforcée par le Mémorial de l'abolition de l'esclavage confié à l'artiste Krzysztof Wodiczko le long du quai de la Fosse où accostaient les navires du commerce triangulaire remontant de Paimboeuf. Le couplage d'héritages (parfois réduits à l'état de trace) à ses œuvres, complète la construction d'une mémoire fondée sur une valorisation patrimoniale plus classique. Cet arrangement spatial et temporel contribue alors à la production territoriale, dans un contexte de renouvellement urbain catalysé par la mise en tourisme de la métropole. La patrimonialisation qui serait en cours et qui n'emprunterait pas forcément la dimension juridique (inscription ou classement sur la liste des Monuments historiques par exemple) relèverait d'un choix politique et s'inscrirait dans la légitimation du projet urbain, comme le montre en abîme la quasi absence de traitement de cet héritage dans d'autres métropoles impliquées pourtant dans le commerce triangulaire comme Le Havre (Gravari-Barbas, 2011), Bordeaux, Saint-Malo ou encore La Rochelle. 


\section{Bibliographie}

Auduc A., 2008. Quand les monuments construisaient la nation, Travaux et documents, $\mathrm{n}^{\circ} 25$, Collection du Comité d'histoire du ministère de la Culture, 640 p.

Austin J.-L., 1970. Quand dire c'est faire, Paris, Seuil, 208 p.

Art actuel, n 43, mars-avril 2006, Yann Kersalé Partiprisme, p. 26.

Barthon C., 2005. Patrimoine industriel ( $\mathrm{xIX}^{\mathrm{e}}-\mathrm{xx}^{\mathrm{e}}$ siècle) et patrimoine $\mathrm{xx}^{\mathrm{e}}$ siècle : Révélateurs d'approches différenciées de la patrimonialisation, l'exemple des villes de l'Ouest français, ESO, Travaux et Documents, n²3, p. 11-16.

Barthon C., Garat I., Gravari-Barbas M., 2007. L'inscription territoriale et le jeu des acteurs dans les événements culturels et festifs : des villes, des festivals, des pouvoirs, Géocarrefour [en ligne], vol. 82/3 [http://geocarrfour.revues/ org/2155].

Bavoux J.-F., 1997. Les littoraux français, Paris, Armand Colin, $268 \mathrm{p}$.

BéGhain P., 1998. Le patrimoine : culture et lien social, Paris, Presses de Sciences Po.

Вогснот C., 2011. Centralités artistiques et recomposition des espaces urbains. Les enjeux d'une géographie de l'art à Paris et à Berlin dans Duhamel Ph., Kadri B. (dir.), Tourisme et mondialisation, Mondes du tourisme, hors série, Éditions Espaces, p. 350-361.

Bossé A., 2012. Visiter pour ne plus voir les choses comme avant, Place Publique, ${ }^{\circ} 31$, p. 53-57.

Brunet R., Ferras R., Théry H., 1993. Article « estuaire », Les mots de la géographie, Paris, Reclus/La Documentation Française, p. 197.

Buissart M., 2009. Patrimoine, artistes et publics, dans Girault-Labalte C., Morice J.-R., Violier Ph. (dir.), Le patrimoine est-il fréquentable?, Presses de l'université d'Angers, p. 189-197.

Chony F., 1992. L'allégorie du patrimoine, Paris, Seuil, 276 p.

Cö̈ffé V., 2011 . Tourisme et «temps mondial ». Jalons pour la lecture d'un monde en mouvement, dans Duhamel Ph., Kadri B. (dir.), Tourisme et mondialisation, Mondes du Tourisme, hors-série, Éditions Espaces, p. 290-298.

Collectif, 2011 , Nantes. L'invention d'une île, Paris, Autrement, coll. «Le Mook», 104 p.

Di Méo G., 1998. Géographie sociale et territoires, Paris, Nathan.

Di MÉo G., 2007. Processus de patrimonialisation et construction des territoires, Actes du colloque Patrimoine et industrie en Poitou-Charentes : connaître pour valoriser, 12-14 octobre, Poitiers-Châtellerault [http://halshs.archivesouvertures.fr/halshs-00281934/en/].

Djian J.-M., 2005. Politique culturelle: la fin d'un mythe, Paris, Gallimard, coll. « Folio actuel », 208 p.

Société publique régionale des Pays de la Loire, 2012. Envies de vacances en Pays de la Loire Service tourisme, Nantes, 48 p.

Florida R., 2002. The Economic Geography of Talent, Annals of the Association of the American Geographers, $\mathrm{n}^{\circ}$ 92(4), p. $743-755$.
Fritsch B., 2006. Nantes-Saint-Nazaire, métropole exemplaire?, L'information géographique, $\mathrm{n}^{\circ}$ 4, p. 25-45.

Gilabert T., 2004. Géographie de l'art contemporain, thèse de doctorat, Université de Nantes.

Gracq J., 1985. La forme d'une ville, Paris, José Corti, 213 p.

Grandet M. et al., 2010. Nantes, la Belle éveillée : le pari de la culture, Toulouse, Éditions de l'attribut, 144 p.

Gravari-Barbas M., 2010. Une patrimonialisation sans appropriation? Le cas de l'architecture de la reconstruction au Havre, Norois, $n^{\circ} 4$, p. 57-73.

Gravari-Barbas M., 2013. Aménager la ville par la culture et le tourisme, Paris, Éditions Le Moniteur, 160 p.

Grésillon B., 2008. Ville et création artistique. Pour une approche de la géographie culturelle, Annales de Géographie, $\mathrm{n}^{\circ}$ 660-661, p. 179-198.

Guide de découverte du patrimoine de l'estuaire de la Loire : L'estuaire vu du flewve, de Nantes à Saint-Nazaire, 84 pages couleurs, 190 illustrations (cartes, aquarelles, photographies).

Heinich N., 2009. La fabrique du patrimoine, Paris, Édition de la Maison des sciences de l'homme, 286 p.

Heinich N., 1993. Du peintre à l'artiste. Artisans et académiciens à l'âge classique, Paris, Minuit, $302 \mathrm{p}$.

Jimenez M., 2005. La Querelle de l'art contemporain, Paris, Gallimard, $237 \mathrm{p}$.

Lazzarotti O., 2003. Article " patrimoine », dans Lévy J., LusSault M. (dir.), Dictionnaire de géographie et de l'espace des sociétés, Paris, Belin, p. 692-693.

Lazzarotti O., Violier Ph. (dir.), 2007. Tourisme et Patrimoine: un moment du Monde, Presses de l'université d'Angers, $246 \mathrm{p}$.

Le Monde, 2 octobre 2010. Décryptage Débats, Murakami à Versailles : audace ou sacrilège?, p. 20-21.

Lussault M., 2009. De la lutte des classes à la lutte des places, Paris, Grasset, 224 p.

Morice J.-R. (coord.), (2009). Population fréquentant la manifestation "Estuaire Nantes-Saint-Nazaire », les machines de l'̂̀le et le Château des ducs de Bretagne à Nantes E Notoriété de la destination Nantes-Saint Nazaire, Commanditaire : Nantes Métropole, Lieu d'édition : université d'Angers - UFR ITBS, 306 p.

Morice J.-R., Violier Ph., 2009. De l'événementiel culturel à la destination touristique : les cas de Lille et Nantes, dans Fagnoni E., Lageiste J. (dir.), L'événementiel et les villes touristiques, Bulletin de l'Association de Géographes Français, $\mathrm{n}^{\circ}$ 3, septembre, Paris, p. 377-387.

Morice J.-R., 2011 . Culture, patrimoine et tourisme par delà les frontières, dans Duhamel Ph., Morvan D., 2009. Entretien avec Daniel Baillon, conseillé pour la culture à la Commission française pour l'Unesco, Ouest-France, 24 avril.

KADRI B. (dir.), Tourisme et mondialisation, hors série, Éditions Espaces, coll. « Mondes du tourisme », p. 205-219.

Nantes Tourisme, guide 2011-2012, Le Voyage à Nantes.

Nantes Passion, été 2009, nº 196.

Nicolas A., 2010. Des espaces témoins du passé au cœur de la fabrique urbaine. Le traitement des patrimoines industriels et portuaires dans les pratiques d'aménagement à Nantes, Lieux communs, dossier "Espaces témoins », $\mathrm{n}^{\circ} 13$, p. 167-184. 
Pirazzoli P., 1997. Les littoraux, Géographie d'aujourd'hui, Paris, Nathan, 191 p.

Place publique, 2007, $\mathrm{n}^{\circ}$ 4, débat Estuaire 2007 : esbrouf ou coup de génie?, [http://revue-placepublique.fr/Sommaires/ Sommaires/Articles/estuaire07.html].

Rapetti D., 2004. Contre vents et marées : « Nantes Atlantique ». La nouvelle donne, M@ppemonde, n 74 [http:// mappemonde.mgm.fr/num2/articles/art04205.html].

Roger A., 1997. Court traité du paysage, Paris, Gallimard, $199 \mathrm{p}$.

ShapIro R., 2004. Qu'est-ce que l'artification?, XVII ${ }^{\text {e Congrès }}$ de l'AISLF, L'individu social, Comité de recherche 18, Sociologie de l'art, Tours, juillet 2004, 9 p.
Veschambre V., 2005. Le recyclage urbain, entre démolition et patrimonialisation : enjeux d'appropriation symbolique de l'espace. Réflexions à partir de quatre villes de l'Ouest, Norois, $\mathrm{n}^{\circ}$ 2, p. 79-92

Vincent E., 2009. Qu'est-ce que la ville créative?, Paris, PUF, $92 \mathrm{p}$.

Volvey A., 2000. L'espace vu du corps, dans LÉVy J., Lussault M. (dir.), Logiques de l'espace, esprit des lieux. Géographies à Cerisy, Paris, Belin, p. 319-332. 